\title{
Structural Characterization of Self-Organized Mono- and Multilayers of Partly Fluorinated Polydialkoxyphosphazenes at the Air/Water Interface
}

\author{
A.I. Buzin ${ }^{1, *}$, G. Brezesinski² ${ }^{2}$ V.S. Papkov ${ }^{3}$, and S.N. Chvalun ${ }^{4,1}$ \\ ${ }^{1}$ Enikolopov Institute of Synthetic Polymer Materials Russian Academy of Sciences, ul. Profsoyuznaya 70, \\ 117393 Moscow, Russia \\ ${ }^{2}$ Max Planck Institute of Colloids and Interfaces, Am Mühlenberg 1, 14476 Potsdam, Germany \\ ${ }^{3}$ Nesmeyanov Institute of Organo-Element Compounds Russian Academy of Sciences, Vavilova ul. 28, \\ 117991 Moscow, Russia \\ ${ }^{4}$ National Research Centre "Kurchatov Institute", Ak. Kurchatova pl. 1, 123182 Moscow, Russia
}

\begin{abstract}
We report studies of the collapse of monolayers of mesomorphic poly[bis-(2,2,3,3,4,4,5,5-octafluoropentoxy)phosphazene] and poly[bis-(2,2,3,3,4,4,5,5,6,6,7,7-dodecafluoroheptoxy)phosphazene]. The structure of the films has been examined at different stages of the collapse process by synchrotron grazing incidence X-ray diffraction. Surface pressure-surface area isotherms show that these films undergo a stepwise collapse with formation of multilayers. Grazing incidence X-ray diffraction reveals only one diffraction peak for the monolayer as well as for multilayers of the two polymers studied, which corresponds to an in-plane interchain spacing $d=15.2 \pm 0.1^{\circ} \AA$ and $17.6 \pm 0.5^{\circ} \AA$, respectively. The in-plane correlation length $\xi_{x y}$ reaches ca. $500 \AA$ for PFP-C5 (33 macromolecules) and ca. $400 \AA$ for PFP-C7 (23 macromolecules), implying a certain mesoscale order similar to a quasi-long-range one.
\end{abstract}

\section{Introduction}

Polyphosphazenes are an important class of organoelement polymers as they possess not only a number of properties useful for technological applications, but also exhibit some interesting structural features [1,2]. Many of these polymers are capable of forming thermotropic mesophases, despite the fact that they possess a flexible main chain and do not contain any mesogenic fragments neither in the main nor in the side chains. These mesophases, named as condis-crystal or hexagonal columnar mesophase, are partly disordered crystalline modifications in thermodynanic equilibrium. They are formed by parallel packed macromolecules in extended macroconformation, main and side chains being conformationally disordered [3, 4]. Similar types of mesophases were observed in some linear and cyclolinear polyorganosiloxanes $[3,4]$.

The phase behavior in bulk of poly[bis-(2,2,3,3-tetrafluoropropoxy)phosphazene] (PFP-C3), poly[bis(2,2,3,3,4,4,5,5-octafluoropentoxy)phosphazene] (PFPC5) and poly[ bis- $(2,2,3,3,4,4,5,5,6,6,7,7$-dodecafluoroheptoxy)phosphazene] (PFP-C7) was studied in Refs. 5 and 6 , which was shown to be determined by an interplay between the packing of macromolecules on the whole and the registering of side groups. All three polymers form low-temperature crystalline phase and condis-mesophase.
PFP-C3 and PFP-C5 also form second crystalline phase, which was interpreted to have a close to hexagonal packing of only side groups. It is an intermediate phase between the low-temperature 3D crystalline and mesomorphic phases. PFP-C7 does not form a similar intermediate phase in bulk.

In this respect the above set of polyphosphazenes with partially fluorinated side alkoxy substituents of different lengths seem to be a suitable object for a more complete understanding the main features of various $2 \mathrm{D}$ and $3 \mathrm{D}$ ordered structures formation affected by the side groups interaction and arising due to consecutive discrete macromolecules layering which is realized at Langmuir monolayers collapse.

Up to date, only a few results were published concerning the behavior of Langmuir monolayers of polyphosphazenes particularly including partly fluorinated ones. Reversible formation of monolayers of poly[bis- $(2,2,2-$ trifluoroethoxy)phosphazene] at the air/water interface, which can be successfully transferred onto solid substrates imparting a very high hydrophobicity to their surface was found in Ref. 7. The layering transitions of ultra-high MW PFP-C3 induced by compression were found to result in the formation of stable multilayers directly at the air/water interface [8]. Their structure was characterized by synchrotron X-ray methods. The

\footnotetext{
* Corresponding author: al37919@gmail.com
} 
transition into a 3D crystalline triclinic phase was observed after formation of the 4-layer thick film.

In this work we present results of the structural investigation of ultrathin layers of PFP-C5 and PFP-C7 performed in situ directly at the air/water interface using synchrotron X-ray diffraction, complementing published earlier corresponding data on PFP-C3 and discuss the generality and differences in the structural changes in Langmuir monolayers of these polymers under increasing surface pressure.

\section{Materials and Methods}

\subsection{Samples}

Poly[ bis-(2,2,3,3,4,4,5,5-octafluoropentoxy)phosphazene] $\left([\eta]=5.45 \mathrm{dL} \mathrm{g} \mathrm{g}^{-1}, M_{\eta}=8.110^{6} \mathrm{Da}\right)$, and poly[ bis(2,2,3,3,4,4,5,5,6,6,7,7-dodecafluoroheptoxy)phosphazene] $\left([\eta]=3.46 \mathrm{dL} \mathrm{g}^{-1}, M_{\eta}=6.810^{6} \mathrm{Da}\right)$ were synthesized in INEOS RAS in the group of Dr. D. R. Tur [6].

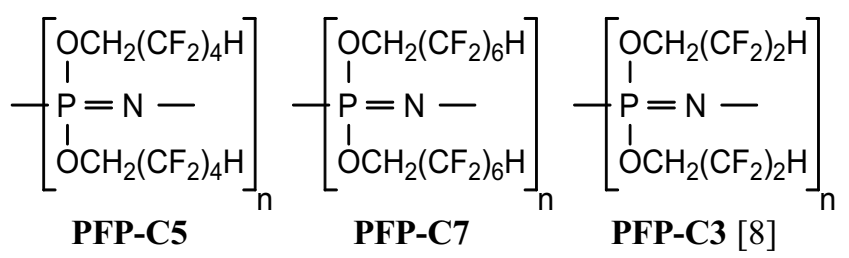

\subsection{Surface pressure-surface area isotherms}

The surface pressure $(\pi) v s$. surface area $(A)$ isotherms were recorded with a R\&K (Potsdam, Germany) trough system. equipped with a Wilhelmy-type pressure sensing system. The velocity of compression was ca. $4 \AA^{2}$ (monomer unit) $)^{-1} \mathrm{~min}^{-1}$. The polymer was spread onto the subphase surface from ethylacetate solution with a typical concentration of $1 \mathrm{mg} \mathrm{mL}^{-1}$. Water used as a liquid subphase was purified with a Millipore desktop system leading to a specific resistance of $18.2 \mathrm{M} \Omega \mathrm{cm}$. The subphase temperature was maintained at $20 \pm 0.5^{\circ} \mathrm{C}$.

\subsection{Synchrotron grazing incidence X-ray diffraction}

Synchrotron grazing incidence X-ray diffraction (GIXD) data collection was performed with a liquid surface diffractometer at the undulator beamline BW1 which was located in the synchrotron radiation research laboratory HASYLAB at DESY (Hamburg, Germany) [9, 10]. GIXD measurements were performed using a Langmuir trough with an area of $480 \mathrm{~cm}^{2}$ placed in an air-tight aluminum container with Kapton windows. Surface pressure was measured with a Wilhelmy filter paper plate with accuracy $\pm 0.2 \mathrm{mN} \mathrm{m}^{-1}$. A polished glass block in the trough reduced the depth of the liquid subphase to $0.3 \mathrm{~mm}$ in the $\mathrm{X}$-ray footprint region and thereby suppressed mechanically excited capillary waves. X-ray experiments were conducted under a water vapor saturated He atmosphere.

GIXD experiments were performed as follows. A monochromatic X-ray beam $(\lambda=1.304 \AA)$ was selected with

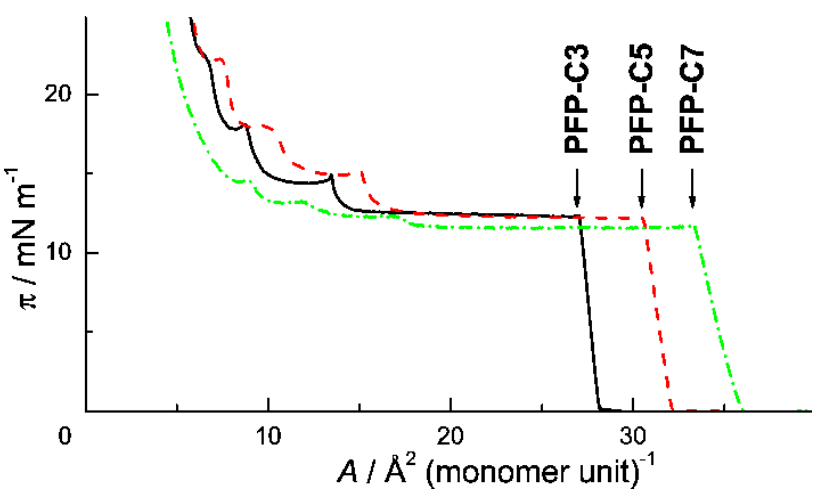

Fig. 1. Surface pressure-surface area isotherms of PFP-C5, PFP-C7 and of PFP-C3 [8] at $20^{\circ} \mathrm{C}$.

a $\operatorname{Be}(002)$ monochromator crystal. The incident grazing angle $\alpha_{i}$ of the X-ray beam was $\sim 0.85 \alpha_{c}$, where $\alpha_{c}=$ $0.129^{\circ}$ is the critical angle for total external reflection from an air/water interface. This limits the penetration depth of the beam to $\sim 8 \mathrm{~nm}$ [9]. Thus, X-ray scattering from the water subphase contributing to the background intensity is efficiently reduced allowing accurate measurements of the X-ray photons diffracted from the monolayer. The dimension of the X-ray footprint on the sample was $2 \mathrm{~mm} \times 50 \mathrm{~mm}$. A linear position sensitive detector (PSD) (OED-100-M, Braun, Garching, Germany) with a vertical acceptance $0<\alpha_{\mathrm{f}}<15^{\circ}$ scanned over $2 \theta_{x y}$ in the range $0<2 \theta_{x y}<40^{\circ}$ was used for recording the diffracted intensity. The measured GIXD data can be visualized as contour plots with the intensity shown as a function of both the horizontal $\left(q_{x y}\right)$ and the vertical $\left(q_{z}\right)$ scattering vector components or projected onto the $q_{x y}$ or $q_{z}$ axes as Bragg peak or Bragg rod data, respectively. The horizontal resolution of $0.008 \AA^{-1}$ was determined by a Soller collimator mounted in front of the PSD. The lattice spacing $d_{h k}$ can be obtained from the inplane diffraction data as $d_{h k}=2 \pi / q_{x y}{ }^{h k}$, where the Bragg peaks have been indexed by the Miller indices $h, k$ to yield the unit cell parameters for the in-plane lattice. The full-width at half-maximum (fwhm) of the Bragg peaks $\Delta q_{x y}$ yields the in-plane correlation length $\xi_{\mathrm{xy}} \approx 0.88 \times 2 \pi /$ $\Delta q_{x y}$. The fwhm of the Bragg rods $\Delta q_{z}$ gives a first estimate of the film thickness: $L \approx 0.88 \times 2 \pi / \Delta q_{z}[11]$.

\section{Results and discussion}

\subsection{General characterization of the $\pi-A$ isotherm}

The polymers investigated are able to form monolayer films on the water surface. Surface pressure-surface area isotherms at $20^{\circ} \mathrm{C}$ of PFP-C5, PFP-C7, as well as of PFP-C3 taken from Ref. 8 for comparison are shown in figure 1. The parameters of the $\pi-A$ isotherms are summarized in table 1. At large areas per monomer unit the surface pressure remains immeasurably low. In this region the condensed monolayer domains coexist with the bare water surface regions. On compression beyond $32.2 \AA^{2}$ (PFP-C5), and $36.0 \AA^{2}$ (PFP-C7) per monomer unit the surface pressure increases linearly up to the beginning of the first plateau. In this region occurs elastic deformation of the dense polymer monolayer of polymer. 
Table 1. Parameters of the $\pi-A$ isotherms of PFP-C5 and PFPC7 ( $n-$ step number).

\begin{tabular}{cccc}
\hline$n$ & $A_{n}\left(\AA^{2}\right)$ & $\pi\left(\mathrm{mN} \mathrm{m}^{-1}\right)$ & $A_{n} / A_{1}$ \\
\hline \multicolumn{4}{c}{ PFP-C5 } \\
1 & 30.5 & 12.2 & \\
2 & 15.2 & 14.9 & 0.5 \\
3 & 10.4 & 17.9 & 0.34 \\
4 & 7.6 & 22.1 & 0.25 \\
\hline \multicolumn{4}{c}{ PFP-C7 } \\
1 & 33.4 & 11.6 & \\
2 & 17.0 & 12.3 & 0.51 \\
3 & 12.0 & 13.2 & 0.36 \\
4 & 9.1 & 14.5 & 0.27 \\
\hline
\end{tabular}

Further compression of the surface layer of the both polymers studied is accompanied by four distinct steps in the surface pressure. The ratios of the surface areas of the steps $A_{n}$ to the surface area of the dense monolayer $A_{1}$ for PFP-C5 are close to $1 / 2,1 / 3$ and $1 / 4$. Such area ratios allow to suggest the formation of the series of discrete multilayers (bilayer, trilayer, tetralayer) similar to that observed earlier in PFP-C3 [8] and mesomorphic cyclolinear polyorganosiloxanes [12-14]. In the case of PFP-C7 the area ratios are slightly overvalued, which may reflect distortion of the shape of the macromolecule in the multilayers (see GIXD data). One can see that PFP-C5 demonstrate about the same surface pressure values as PFP-C3 and only possesses larger molecular area due to longer side groups. At the same time, PFP-C7 shows lower surface pressure of the first step as well as average surface pressure of the following steps ca. $1 \mathrm{mN} \mathrm{m}^{-1}$ vs. $3-4 \mathrm{mN} \mathrm{m}^{-1}$, which is typical for PFPC3 and PFP-C5. This may indicate lower stability of the PFP-C7 layer in comparison with PFP-C3 and PFP-C5.

It is important to note that the surface pressure-surface area isotherms are not equilibrium ones. On decompression of the multilayer the surface pressure drops to a value slightly lower than the pressure of the first plateau and stays constant. The surface pressure decreases after stopping the compression and increases after stopping the expansion. An equillibrium surface pressure value exists for every plateau region. The reversibility and stability of the $\pi-A$ isotherm of PFP-C3 was discussed in detail in Ref. 8. PFP-C5 and PFP-C7 show similar behavior.

\subsection{Structure of mono- and multilayers}

Partly fluorinated polydialkoxyphosphazenes demonstrate the thermotropic polymorphism, i.e., the 3D low-temperature and intermediate crystalline phases and one mesophase form in the bulk state $[5,6]$. They transform reversibly one into another on heating and cooling. In PFPC3 the crystal-crystal transition occurs in a temperature range from 5 to $30^{\circ} \mathrm{C}$ depending on the thermal history of the sample, whereas the crystal-mesophase transition occurs above $80^{\circ} \mathrm{C}$. In PFP-C5 these two transitions are observed at lower temperatures $\left(8^{\circ} \mathrm{C}\right.$ and $45^{\circ} \mathrm{C}$, respecttively). PFP-C7 on heating transforms from low-temperature crystalline phase directly into mesophase at $0{ }^{\circ} \mathrm{C}$. The low-temperature crystalline Phase I is characterized by the common crystalline lattice for the main chain and side chains packed into a monoclinic unit cell. The inter-
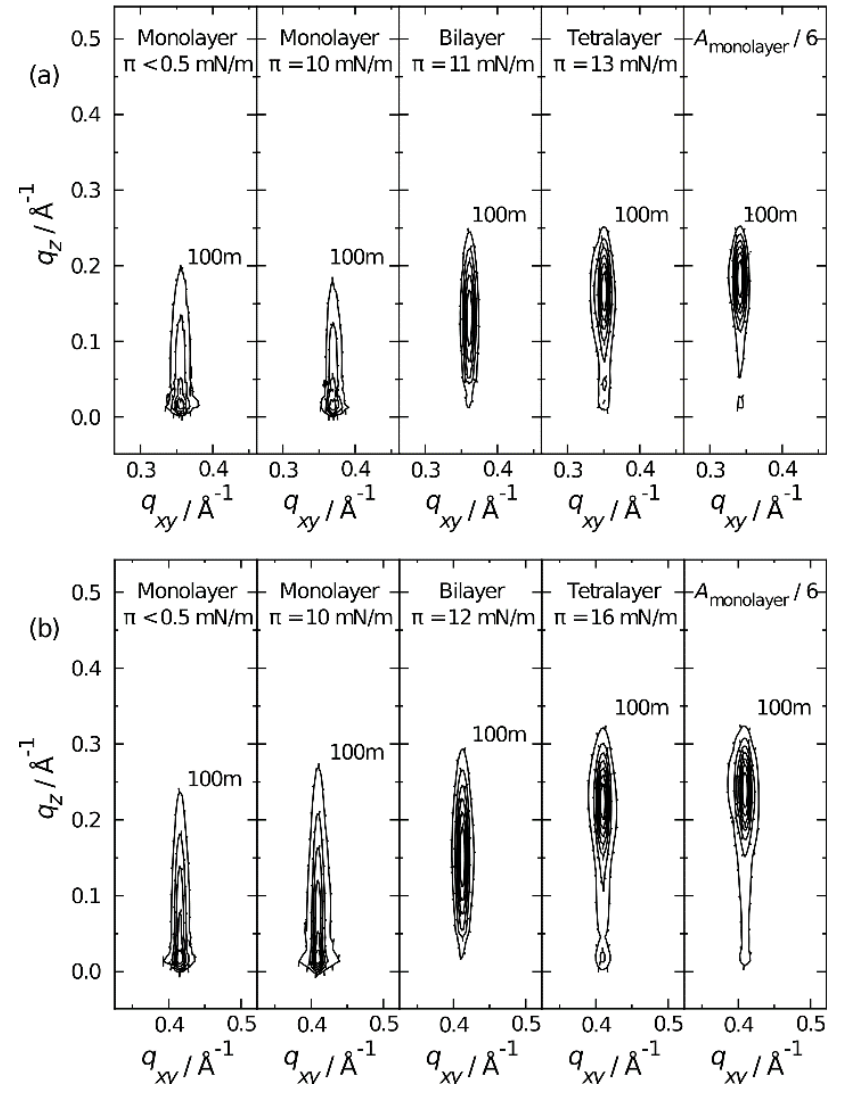

Fig. 2. Contour plots of the scattered intensity as a function of the in-plane $q_{x y}$ and out-of-plane $q_{z}$ scattering vector components of PFP-C5 (a) and PFP-C7 (b) mono-, bi-, tetralayers at various surface pressures (indicated) as well as at $1 / 6$ of the dense monolayer area.

mediate crystalline Phase II is controlled mainly by close to hexagonal packing of the side chains. The transition into the mesomorphic phase is accompanied by a distortion of the side chains packing. Only regular packing of the main chains of macromolecules in the plane perpendicular to their axes remains in the mesomorphic state.

Selected GIXD data for thin films of PFP-C5 and PFP-C7 are shown in figure 2 as 2D contour plots. The scattering from the monolayer shows only a single Bragg peak related to the in-plane interchain distance. The absence of any further diffraction peaks, and therefore, the absence of any long-range correlation along the macromolecules both in monolayers, and in the collapsed films, can most likely be explained as a consequence of a conformational disorder and/or non-registering of one polymer chain along the next one, that is typical for the condis-mesophase in the bulk. In-plane repeating distance $d$ and correlation length $\xi$ as well as layer thickness $L$ obtained from the GIXD experiments for the monolayers and the collapsed mesomorphic films of PFP-C5, PFP-C7 and for comparison of PFP-C3 [8] are shown in figure 3.

Monolayers exhibit a single diffraction peak already at a very low surface pressure (less than $0.5 \mathrm{mN} \mathrm{m}^{-1}$ ) just before the beginning of the first increase in $\pi$. The position of the Bragg peak gives an in-plane repeating distance $d$ in the monolayer $(d=15.2 \AA$ for PFP-C5 and $17.6 \AA$ for PFP-C7). These values are slightly lower than the interchain distances $(d=15.7 \AA$ and $18.1 \AA$, respecti- 

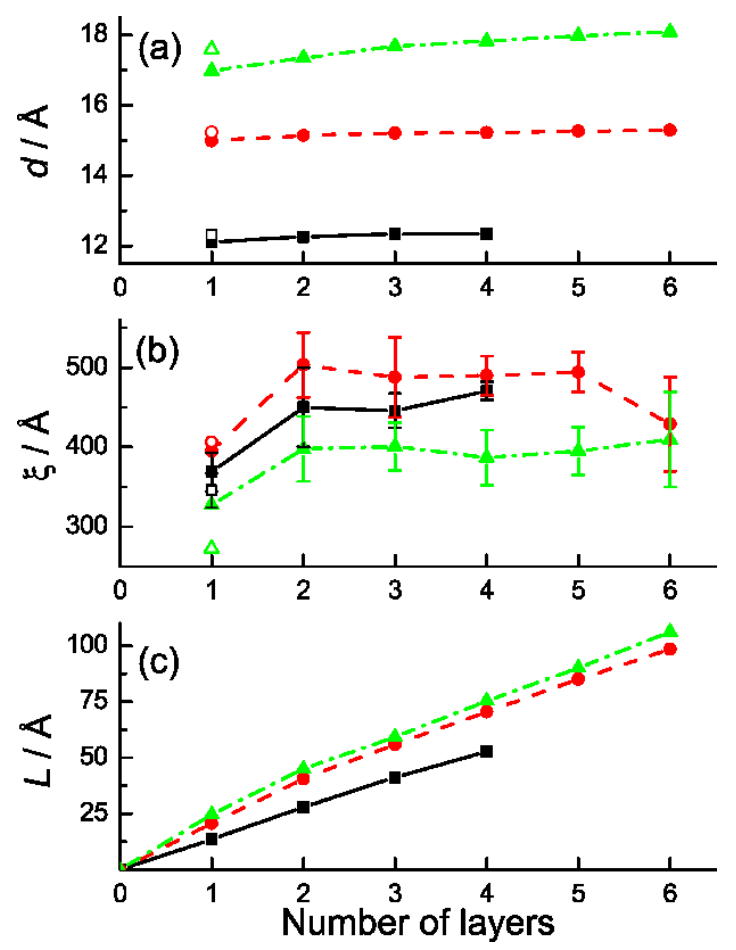

Fig. 3. In-plane repeating distance $d$ and correlation length $\xi$ as well as layer thickness $L$ obtained from the GIXD experiments for the mesomorphic surface layers of PFP-C5 (circles), PFP-C7 (triangles) and PFP-C3 (squares) [8]. Open symbols indicate points measured at $\pi<0.5 \mathrm{mN} \mathrm{m}^{-1}$. Errors in $d$ and $L$ are smaller than symbol size.

vely) measured in the bulk condis-mesophase with hexagonal columnar packing of the main chains [6]. Compression of the monolayer is accompanied by a slight decrease in the repeating distance (at $10 \mathrm{mN} \mathrm{m}^{-1} d=15.0 \AA$ and $17.0 \AA$, respectively). With increasing number of layer, the in-plane repeating distance of PFP-C5 stays constant at $c a$. $15.2 \AA$, while the in-plane repeating distance of PFP-C7 grows reaching $18.1 \AA$ for $1 / 6$ of the dense monolayer area. Analysis of the observed fwhm of the Bragg peak $\Delta q_{x y}$ yields the in-plane correlation length $\xi_{x y}$, i.e., the extent of lateral positional correlations within the monolayer and collapsed films, perpendicular to the axes of the macromolecules. Starting from bilayer the correlation length reaches $c a$. $500 \AA$ for PFP-C5 (33 macromolecules) and ca. $400 \AA$ for PFP-C7 (23 macromolecules). The analysis of fwhm of the Bragg rod allows estimating the thickness $L$ of the mono- and multilayers. The monolayer thickness is $20.7 \AA$ for PFP-C5 and $24.6 \AA$ for PFP-C7, thus indicating essential distortion of the cylindrical shape of the macromolecules in the monolayer. With increasing layer numbers, the layer thickness grows nonlinearly. At $1 / 6$ of the dense monolayer area $L=98.3 \AA$ for PFP-C5 and 106.1 $\AA$ for PFP-C7, that means approaching the cylindrical shape of the macromolecules, which is typical for bulk hexagonal columnar mesophase.

\section{Conclusion}

We investigated structure of mono- and multilayers of mesomorphic partly fluorinated polydialkoxyphosphazenes at the air/water interface by GIXD. The scattering from both the monolayer and the multilayers shows only a single Bragg peak, which indicates regular packing in the direction perpendicular to the axes of the macromolecules. The macromolecules are mainly in extended chain conformation and their main chains are aligned parallel to each other. The periodicity along the macromolecules is largely disrupted due to the conformational disorder and non-registering in the main chains, that is typical for the condis-mesophase in the bulk state. The extent of lateral correlations between parallel macromolecules ranges from 33 (for PFP-C5) to 23 (for PFP-C7) interchain distances, demonstrating a mesoscale-range order for the collapsed films, which resembles the $2 \mathrm{D}$ order in the columnar mesophase. The monolayer collapse of polyphosphazenes studied looks similar to that observed earlier for some cyclolinear polyorganosiloxanes [12] suggesting that the ability of organo-element polymer to form columnar mesophase can be a prerequisite of the phenomenon of multilayer formation.

\section{Acknowledgements}

This work was supported by Russian Science Foundation (project 14-13-01402). We are grateful to HASYLAB at DESY, Hamburg, Germany for beam time.

\section{References}

1. H.R. Allcock, Chemistry and applications of polyphosphazenes (Wiley-Interscience, 2003)

2. A.K. Andrianov, Polyphosphazenes for biomedical applications (John Wiley \& Sons, 2009)

3. Y.K. Godovsky, V.S. Papkov, Adv. Polym. Sci. 88, 129 (1989)

4. G. Ungar, Polymer 34, 2050 (1993)

5. D.Y. Tsvankin, M.V. Gerasimov, V.P. Zhukov, I.I. Dubovik, D.R. Tur, V.S. Papkov, J. Polym. Sci., Part B: Polym. Phys. 30, 851 (1992)

6. M.V. Gerasimov, Phase transitions and structure of ordered phases in partly fluorinated polydialkoxyphosphazenes, $\mathrm{Ph}$. D. Thesis (in Russian) (Moscow, INEOS RAS, 1994)

7. L.L. Mack, R.J. Fitzpatrick, H.R. Allcock, Langmuir 13, 2123 (1997)

8. A.I. Buzin, G. Brezesinski, D.R. Tur, V.S. Papkov, A.V. Bakirov, S.N. Chvalun, Macromolecules 48, 3327 (2015)

9. J. Als-Nielsen, D. Jacquemain, K. Kjær, L. Franck, M. Lahav, L. Leiserowitz, Phys. Rep. 246, 251 (1994)

10. R. Frahm, J. Weigelt, G. Meyer, G. Materlik, Rev. Sci. Instrum. 66, 1677 (1995)

11. K. Kjær, Phys. B 198, 100 (1994)

12. S.I. Belousov, A.I. Buzin, Y.K. Godovskii, Vysokomol. Soedin., Ser. B 41, 1687 (1999)

13. T.R. Jensen, K. Kjær, G. Brezesinski, J. Ruiz-Garcia, H. Möhwald, N.N. Makarova, Y.K. Godovsky, Macromolecules 36, 7236 (2003)

14. Y.K. Godovsky, G. Brezesinski, J. Ruiz-Garcia, H. Möhwald, T.R. Jensen, K. Kjær, N.N. Makarova, Macromolecules 37, 4872 (2004) 